NASA Technical Memorandum 106197

ICOMP-93-18; CMOTT-93-07

\title{
A Numerical Study of Confined Turbulent Jets
}

J. Zhu and T.-H. Shih

Institute for Computational Mechanics in Propulsion and

Center for Modeling of Turbulence and Transition

Lewis Research Center

Cleveland, Ohio

Prepared for the

Winter Annual Meeting sponsored by the American Society of Mechanical Engineers

New Orleans, Louisiana, November 28-December 3, 1993

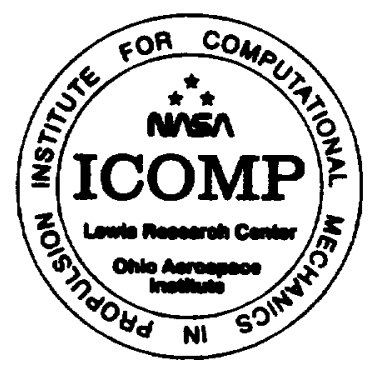





\title{
A NUMERICAL STUDY OF CONFINED TURBULENT JETS
}

\author{
J. Zhu and T.-H. Shih \\ Institute for Computational Mechanics in Propulsion \\ and Center for Modeling of Turbulence and Transition \\ NASA Lewis Research Center \\ Cleveland, Ohio 44135
}

\begin{abstract}
A numerical investigation is reported of turbulent incompressible jets confined in two ducts, one cylindrical and the other conical with a $5^{\circ}$ divergence. In each case, three Craya-Curtet numbers are considered which correspond, respectively, to flow situations with no, moderate and strong recirculation. Turbulence closure is achieved by using the $K-\varepsilon$ model and a recently proposed realizable Reynolds stress algebraic equation model that relates the Reynolds stresses explicitly to the quadratic terms of the mean velocity gradients and ensures the positiveness of each component of the turbulent kinetic energy. Calculations are carried out with a finite-volume procedure using boundary-fitted curvilinear co-ordinates. A second-order accurate, bounded convection scheme and sufficiently fine grids are used to prevent the solutions from being contaminated by numerical diffusion. The calculated results are compared extensively with the available experimental data. It is shown that the numerical methods presented are capable of capturing the essential flow features observed in the experiments and that the realizable Reynolds stress algebraic equation model performs much better than the $K-\varepsilon$ model for this class of flows of great practical importance.
\end{abstract}

\section{Introduction}

This paper is concerned with numerical computations of confined turbulent jets. The general features of the flow under consideration are sketched in Fig.1. At the entrance, two uniform flows, a jet of high velocity and an ambient stream of low velocity, are discharged into the duct. Due to turbulent entrainment, the jet increases its mass flux while spreading and this causes an equal decrease in the mass flux of the ambient stream. An adverse pressure gradient is thus set up by the decrease in the velocity of the ambient stream which can be considered as a 
potential flow. When the ratio of jet to ambient velocities at the entrance is above a critical value, a recirculation zone occurs at the duct wall downstream of the inlet plane. This is because the jet has consumed the whole ambient flow before reaching the wall and further entrainment must create reverse flows in order to maintain the total mass flux conservation. For a given geometry, recirculation as well as adverse pressure gradients can be intensified by increasing the jet to ambient velocity ratio at the entrance. These features are common to many flows involving two streams of different velocities in industrial apparatus, in particular, in combustion chambers in which recirculating currents are used to enhance flame mixing and stability, and in ejectors whose role as a jet pump is achieved through the pressure rise generated by the jet entrainment. Therefore, the understanding of confined jet flows is of great practical importance to the design of such devices.

A large amount of theoretical and experimental research has been devoted to confined jets (Rajaratnam, 1976). Early theoretical studies were restricted to the development of integral methods based on similarity assumptions (Craya and Curtet, 1955, Curtet, 1960, Hill, 1967 and 1973, Mikhail 1960 and Razinsky and Brighton, 1972). The essential features of these methods are presented in detail in Rajaratnam (1976). Among the most well-known of them is the approximate confined jet theory of Craya and Curtet. They found that confined jets in constant area ducts can form a family characterized by a single non-dimensional parameter $C_{t}$ termed the CrayaCurtet number, provided that the flows remain self-similar. This condition cannot be satisfied in general, especially in the presence of recirculation, and the CrayaCurtet number is not constant in variable area ducts. Nevertheness, the values of $C_{t}$ at the entrance remain useful to characterize the inlet flow conditions. Experimental studies can be found in Barchilon and Curtet (1964), Barchilon (1969), Binder and Kian (1983), Kian (1981) and Razinsky and Brighton (1971). Experiments show that recirculation occurs in cylindrical ducts when $C_{t} \leq 0.96$ and in a conical duct with a $5^{\circ}$ divergence when $C_{t} \leq 1.1$.

The general applicability of the classical integral method is invalidated by the complexities of the flow. At the present time, the only feasible theoretical solution is to use the differential method that solves the complete governing equations with the aid of turbulence modeling. Several authors (Gosman et al., 1979, Habib and Whitelaw, 1979 and 1981, Jones and Marquis, 1985, Khalil et al., 1975 and Zhu, 1986) have reported the relevant calculations using such differential methods. In these calculations, turbulence effects were represented either by the $K-\varepsilon$ model or by second-moment closures. The success of the differential methods depends, to a large extent, on the performance of the turbulence model used. These numerical studies showed that the calculations with the $K-\varepsilon$ model could reproduce some 
basic flow characteristics observed in the experiments in general, but large discrepances were present in detail. These discrepances were mostly attributed to inherent imperfections of the model such as the isotropic eddy-viscosity representation and the insensitivity to streamline curvature effects. Second-moment closures have no such drawbacks and represent a higher level of turbulence modeling beyond the eddy-viscosity level. However, it was found in a systematical numerical study (Zhu, 1986) that second-moment closures, whether of the algebraic or of the transport form, did not manifest themselves to be significantly superior to the $K-\varepsilon$ model in the computation of the confined jets. It should be pointed out that the previous calculations were almost exclusively performed on coarse grids and with the hybrid (central/upwind differencing) scheme (Spalding, 1972) that is essentially of firstorder accuracy in convection-dominated situations. Recent work of Zhu and Rodi (1992) has shown that the calculations of confined jets were very sensitive to the approximation of the convection terms of transport equations and the hybrid scheme could not yield grid-independent results, especially for the turbulent quantities, on a grid as fine as $102 \times 82$ points. Therefore, it is certain that the early calculations were contaminated to some extent by numerical diffusion and the performance of the turbulence models for the confined jet flows needs to be re-assessed on a numerically reliable basis.

Recently, Shih and Lumley (1993) have shown that the Reynolds stress, being a second rank tensor, can be expressed as a fourth-order polynomial of the mean velocity gradients. This is the most general stress-strain relationship within the framework of algebraic turbulence modeling, with the linear stress-strain relation in the Boussinesq's eddy-viscosity concept being its first-order approximation. Based on this, Shih et al. (1993) have recently proposed a quadratic stress-strain relation in conjunction with the two modeled equations of $K$ and $\varepsilon$ for practical calculations. Realizability constraints (Lumley, 1978 and Schumann, 1977) have been used to derive appropriate functional expressions for the model coefficients, in particular, the coefficient $C_{\mu}$ has been naturally related to the time scale ratio of the turbulence to the mean strain rate. As a result, the model ensures the positivity of individual turbulent normal stresses, an important feature that is not present in most existing turbulence models. The empirical constants in the model have been fine-tuned in the calculation of two backward-facing step flows. As the model appears promising as a competitive alternative in the turbulence modeling arsenal, it is of interest to test its performance for confined jets which, due to their complicated flow structures such as strong recirculation coupled with severe adverse pressure gradients, constitute a challenge to the turbulence modeling.

To this end, calculations are performed for the flows studied experimentally by 
Barchilon and Curtet (1964) in a cylindrical duct and by Binder and Kian (1983) in a conical duct with $5^{\circ}$ divergence. These two experiments have been chosen in this study because of their diverse flow conditions which vary gradually from no to strong recirculation. The standard $K-\varepsilon$ model is also used, since it is the most popular turbulence model used today in calculations of complicated flows and can also be used to highlight the performance of the new model. The numerical accuracy of solutions is ensured by using a second-order accurate, bounded convection scheme (Zhu, 1991a) and sufficiently fine grids. The performances of the models are examined through extensive comparisons with experimental data.

\section{Calculation Approach}

2.1 Turbulence Models. Incompressible turbulent flows are governed by the statistically averaged continuity and Navier-Stokes equations. The Reynolds or turbulent stresses $\tau_{i j}\left(=-\rho \overline{u_{i} u_{j}}\right)$ appearing in these equations are calculated by using the following two models:

1) $K-\varepsilon$ model (Launder and Spalding, 1974)

$$
\begin{aligned}
& \tau_{i j}=\mu_{t}\left(U_{i, j}+U_{j, i}\right)-\frac{2}{3} \rho K \delta_{i j} \\
& \mu_{t}=C_{\mu} \frac{\rho K^{2}}{\varepsilon}, \quad C_{\mu}=0.09
\end{aligned}
$$

where the turbulent kinetic energy $K$ and its dissipation $\varepsilon$ are calculated, respectively, by the modeled transport equations given in Table 1.

2) Realizable Reynolds stress algebraic equation (RRSAE) model (Shih et al., 1993)

$$
\begin{gathered}
\tau_{i j}=\mu_{t}\left(U_{i, j}+U_{j, i}\right)+T_{i j}-\frac{2}{3} \rho K \delta_{i j} \\
\mu_{t}=C_{\mu} \frac{\rho K^{2}}{\varepsilon}, \quad C_{\mu}=\frac{2 / 3}{A_{1}+\eta+\gamma \xi}
\end{gathered}
$$

where

$$
\begin{aligned}
T_{i j}= & -\frac{\rho K^{3}}{\left(A_{2}+\eta^{3}\right) \varepsilon^{2}}\left[C_{\tau 1}\left(U_{i, k} U_{k, j}+U_{j, k} U_{k, i}-\frac{2}{3} \Pi \delta_{i j}\right)\right. \\
& \left.+C_{\tau 2}\left(U_{i, k} U_{j, k}-\frac{1}{3} \tilde{\Pi} \delta_{i j}\right)+C_{\tau 3}\left(U_{k, i} U_{k, j}-\frac{1}{3} \tilde{\Pi} \delta_{i j}\right)\right]
\end{aligned}
$$




$$
\begin{gathered}
\Pi=U_{k, l} U_{l, k}, \quad \tilde{\Pi}=U_{k, l} U_{k, l} \\
\eta=\frac{K S}{\varepsilon}, \quad S=\left(2 S_{i j} S_{i j}\right)^{1 / 2}, \quad S_{i j}=\frac{1}{2}\left(U_{i, j}+U_{j, i}\right) \\
\xi=\frac{K \Omega}{\varepsilon}, \quad \Omega=\left(2 \Omega_{i j}^{*} \Omega_{i j}^{*}\right)^{1 / 2}, \quad \Omega_{i j}^{*}=\left(U_{i, j}-U_{j, i}\right) / 2+4 \varepsilon_{m j i} \omega_{m}
\end{gathered}
$$

$\omega_{m}$ is the rotation rate of the reference frame, and the model constants are

$$
C_{\tau 1}=-4, \quad C_{\tau 2}=13, \quad C_{\tau 3}=-2, \quad A_{2}=1000
$$

In the work of Shih et al. (1993) and Zhu and Shih (1993), the following two sets of values for $A_{1}, \gamma$ have been tested

$$
\begin{array}{cc}
A_{1}=5.5, & \gamma=0 \\
A_{1}=1.25, & \gamma=0.9
\end{array}
$$

and both were found to give almost identical predictions for the two backward-facing step flows. With Eq.( 11$)$, the rotational effect of the mean flow explicitly enters into $C_{\mu}$. However, the values in Eq. (10) are taken in the present work. The $K$ and $\varepsilon$ in the RRSAE model are calculated with the same equations as in the $K-\varepsilon$ model. It can be seen that the RRSAE model reduces to the $K-\varepsilon$ model if the quadratic terms $T_{i j}$ are set to zero.

2.2 Curvilinear Form of Governing Equations. Boundary-fitted curvilinear co-ordinates are used in order to handle irregular flow boundaries. In such coordinates, the two-dimensional, steady-state governing equations using Cartesian velocity components $U_{i}$ can be written in the following general form:

$$
\left(C_{i} \phi-D_{\mathbf{i} \phi}\right)_{, x_{i}}=r^{\alpha} J S_{\phi}, \quad i=1,2
$$

where the convective coefficients $C_{i}$, the diffusion terms $D_{i \phi}$, and the source terms $S_{\phi}$ are given in Table 1 for different dependent variables $\phi ; J$ is the Jacobian of the transformation between the curvilinear coordinates $x_{i}$ and the reference Cartesian coordinates $y_{i}$. Eq. $(12)$ includes both the plane $(\alpha=0)$ and axisymmetric $(\alpha=1)$ forms. In the latter form, $y_{1}(=x)$ and $y_{2}(=r)$ are in the axial and radial directions, respectively.

In the curvilinear transformation, Eqs. (1) - ( 8) all remain the same, while the velocity gradients are calculated as follows: 


$$
\begin{aligned}
& U_{1,1}=\left(\beta_{1}^{1} U_{1, x_{1}}+\beta_{1}^{2} U_{1, x_{2}}\right) / J \\
& U_{1,2}=\left(\beta_{2}^{1} U_{1, x_{1}}+\beta_{2}^{2} U_{1, x_{2}}\right) / J \\
& U_{2,1}=\left(\beta_{1}^{1} U_{2, x_{1}}+\beta_{1}^{2} U_{2, x_{2}}\right) / J \\
& U_{2,2}=\left(\beta_{2}^{1} U_{2, x_{1}}+\beta_{2}^{2} U_{2, x_{2}}\right) / J \\
& U_{3,3}=\alpha U_{2} / r
\end{aligned}
$$

\begin{tabular}{|c|c|c|c|}
\hline$\phi$ & $D_{1 \phi}$ & $D_{2 \phi}$ & $S_{\phi}$ \\
\hline 1 & 0 & 0 & 0 \\
\hline$U_{1}$ & $\begin{array}{c}\frac{r^{\alpha} \Gamma_{1}}{J}\left(D_{1}+\beta_{1}^{1} \omega_{1}^{1}+\beta_{2}^{1} \omega_{1}^{2}\right) \\
\quad+r^{\alpha}\left(\beta_{1}^{1} T_{11}+\beta_{2}^{1} T_{12}\right)\end{array}$ & $\begin{array}{c}\frac{r^{\alpha} \Gamma_{1}}{J}\left(D_{2}+\beta_{1}^{2} \omega_{1}^{1}+\beta_{2}^{2} \omega_{1}^{2}\right) \\
+r^{\alpha}\left(\beta_{1}^{2} T_{11}+\beta_{2}^{2} T_{12}\right)\end{array}$ & $-\frac{1}{J}\left(\beta_{1}^{1} P_{, x_{1}}+\beta_{1}^{2} P_{, x_{2}}\right)$ \\
\hline$U_{2}$ & $\begin{array}{c}\frac{r^{\alpha} \Gamma_{2}}{J}\left(D_{1}+\beta_{1}^{1} \omega_{2}^{1}+\beta_{2}^{1} \omega_{2}^{2}\right) \\
+r^{\alpha}\left(\beta_{1}^{1} T_{21}+\beta_{2}^{1} T_{22}\right)\end{array}$ & $\begin{array}{c}\frac{r^{\alpha} \Gamma_{2}}{J}\left(D_{2}+\beta_{1}^{2} \omega_{2}^{1}+\beta_{2}^{2} \omega_{2}^{2}\right) \\
\quad+r^{\alpha}\left(\beta_{1}^{2} T_{21}+\beta_{2}^{2} T_{22}\right)\end{array}$ & $\begin{array}{r}-\frac{1}{J}\left(\beta_{2}^{1} P_{, x_{1}}+\beta_{2}^{2} P_{, x_{2}}\right) \\
-\alpha\left(2 \Gamma_{2} \frac{U_{2}}{r^{2}}+\frac{T_{33}}{r}\right)\end{array}$ \\
\hline$K$ & $\frac{r^{\alpha} \Gamma_{3}}{J} D_{1}$ & $\frac{r^{\alpha} \Gamma_{3}}{J} D_{2}$ & $G-\rho \epsilon$ \\
\hline$\epsilon$ & $\frac{r^{\alpha} \Gamma_{4}}{J} D_{1}$ & $\frac{r^{\alpha} \Gamma_{4}}{J} D_{2}$ & $\left(C_{1 \epsilon} G-C_{2 \epsilon} \rho \epsilon\right) \frac{\epsilon}{K}$ \\
\hline & $\begin{array}{l}C_{1}=\rho r^{\alpha}\left(\beta_{1}^{1} U_{1}+\beta_{2}^{1} U_{2}\right) \\
D_{1}=B_{1}^{1} \phi_{, x_{1}}+B_{2}^{1} \phi_{, x_{2}} \\
\beta_{j}^{i}=\text { cofactor of } y_{j, x_{i}} \text { in } \\
B_{j}^{i}=\beta_{1}^{i} \beta_{1}^{j}+\beta_{2}^{i} \beta_{2}^{j}, \quad \omega_{j}^{i} \\
P=p+\frac{2}{3} \rho K \quad(p \text { is the } \\
\Gamma_{n}=\mu+\frac{\mu_{t}}{\sigma_{n}}, \quad(n=1 \\
G=\tau_{11} U_{1,1}+\tau_{22} U_{2,2}+c \\
C_{1 \epsilon}=1.44, C_{2 \epsilon}=1.92,\end{array}$ & $\begin{array}{l}\quad C_{2}=\rho r^{\alpha}\left(\beta_{1}^{2} U_{1}+\beta_{2}^{2} U_{2}\right) \\
D_{2}=B_{1}^{2} \phi_{, x_{1}}+B_{2}^{2} \phi_{, x_{2}} \\
J \\
=\beta_{j}^{1} U_{i, x_{1}}+\beta_{j}^{2} U_{i, x_{2}}, \\
\text { mean static pressure }) \\
2,3,4) \\
\chi \tau_{33} U_{3,3}+\tau_{12}\left(U_{1,2}+U_{2,1}\right) \\
\sigma_{1}=\sigma_{2}=\sigma_{3}=1, \sigma_{4}=1.3\end{array}$ & $=\mid \begin{array}{ll}y_{1, x_{1}} & y_{2, x_{1}} \\
y_{1, x_{2}} & y_{2, x_{2}}\end{array}$ \\
\hline
\end{tabular}

where $U_{j, x_{i}}$ is the partial derivative of $U_{j}$ with respect to $x_{i}$.

Table 1. Individual terms in the transport equations 
2.3 Boundary Conditions. Computational boundaries involved are inlet, outlet, axis of symmetry and solid wall. Among them, the inlet conditions require special attention because they have a considerable influence on the calculations (Zhu et al., 1987 and Zhu and Rodi, 1992). At the inlet, the jet and the ambient flow have uniform velocities $U_{J}$ and $U_{a}$ (Fig.1), but the flow conditions in the initial shear layer between the jet and the ambient flow are unknown and determination of these conditions is not a trivial matter. In the present work, the parabolic entrance region (PER) scheme of Zhu et al. (1987) is used. The PER scheme was developed on the assumption that although the flow as a whole is elliptic, there is a short region near the entrance where the flow is parabolic. A parabolic calculation is first carried out over a short distance between $x=0$ and $x=x_{e}$ by using the following mixing length model

$$
\rho \overline{u v}=-\mu_{t} U_{, r}, \quad \mu_{t}=C^{2}\left(r_{2}-r_{1}\right)^{2}\left|U_{, r}\right|
$$

where $U\left(=U_{1}\right)$ is the axial mean velocity, $r_{1}$ and $r_{2}$ are the coordinates of the inner and outer edge of the initial shear layer (Fig.1) and $C$ is an empirical coefficient derived from the experiment of Rajaratnam and Pani (1972)

$$
C^{2}=0.0042+0.004 U_{a} / U_{J}, \quad 0 \leq U_{a} / U_{J} \leq 0.2
$$

The results of the parabolic calculation are then used as the inlet conditions at $x=x_{e}$ for the elliptic calculation. At this location, the inlet values of $K$ and $\varepsilon$ are calculated by

$$
K=-\overline{u v} / 0.3, \quad \varepsilon=0.09 \rho K^{2} / \mu_{t}
$$

It was found $(\mathrm{Zhu}, 1986)$ that the PER scheme gives satisfactory predictions in the parabolic entrance region and that the elliptic calculations are insensitive to $x_{e}$ provided that $1 \leq x_{e} / d_{o} \leq 3$.

The outlet boundary is placed at $x=10 D_{0}$ which is sufficiently far away from the main region of interest. At this boundary streamwise gradients of all variables are set to zero. Along the axis of symmetry the normal velocity component and the normal gradients of the other variables are set to zero. The standard wall-function approach (Launder and Spalding, 1974) is used to bridge the viscous sublayer at the duct wall.

2.4 Numerical Procedure. The system of transport equations (12) is solved by using a conservative finite-volume method designed for calculating incompressible elliptic flows with complex boundaries. The method uses a non-staggered variable 
arrangement, namely, all the dependent variables are stored at the geometric center of each control volume. The momentum interpolation of Rhie and Chow (1983) is used to avoid checkerboard oscillations usually associated with non-staggered grids. The velocity-pressure coupling is achieved via the SIMPLEC algorithm (Van Doormal and Raithby, 1984). Numerical implementation of the RRSAE model is straightforward; the linear part is treated in the same manner as in the $K-\varepsilon$ model and the quadratic part is included in the source terms. To ensure numerical accuracy and stability, the convection terms of all the transport equations are differenced by the hybrid linear/parabolic approximation (HLPA) of second-order accuracy (Zhu, 1991a), and all the other terms by the conventional central differencing scheme. The resulting set of algebraic difference equations is solved with the strongly implicit procedure of Stone (1968). The iterative solution process is regarded as converged when the maximum normalized residue of all the dependent variables is below $0.5 \%$. The details of the present numerical procedure are given in Rodi et al. (1989) and Zhu (1991b).

\section{Results}

The experiments of Barchilon and Curtet (1964) and Binder and Kian (1983) were taken as the test cases, hereinafter termed BC- and BK-case, respectively. The geometrical dimensions and the inlet flow conditions of both cases are given in Tables 2 and 3.

Table 2. Geometrical dimensions of the ducts

\begin{tabular}{ccccc}
\hline case & $D_{\circ}(\mathrm{cm})$ & $d_{\circ}(\mathrm{cm})$ & $\theta($ degree $)$ & $L(\mathrm{~cm})$ \\
\hline BC & 16 & 1.2 & 0 & \\
\hline BK & 16 & 1.6 & 2.5 & 64 \\
\hline
\end{tabular}

In the BK-case, the velocity field and the pressure field were measured separately using the different inlet velocities as shown in Table 3. The Craya-Curtet number $C_{t}$ is defined by

$$
C_{t}=\frac{1}{\sqrt{m}}, \quad m=\frac{S}{Q^{2}} \int_{S}\left(U^{2}+\frac{p}{\rho}\right) d S-\frac{1}{2}
$$

where $m$ is the total momentum flux non-dimensionalized with the total flow rate $Q$ and the duct area $S$. 
Table 3. Inlet flow conditions

\begin{tabular}{cccc}
\hline case & $C_{t}$ & $U_{J}(\mathrm{~cm} / \mathrm{s})$ & $U_{a}(\mathrm{~cm} / \mathrm{s})$ \\
\hline \multirow{2}{*}{$\mathrm{BC}$} & 0.976 & 1293.6 & 84.48 \\
\cline { 2 - 4 } & 0.506 & 1253.8 & 39.81 \\
\cline { 2 - 4 } & 0.152 & 1296.2 & 7.42 \\
\hline BK & 1.230 & 40 & 4.885 \\
\cline { 2 - 4 } $\begin{array}{c}\text { velocity } \\
\text { field) }\end{array}$ & 0.775 & 40 & 2.662 \\
\cline { 2 - 4 } & 0.590 & 40 & 2.330 \\
\hline BK & 1.090 & 650 & 61.38 \\
\cline { 2 - 4 } (pressure & 0.842 & 650 & 46.58 \\
\cline { 2 - 4 } field) & 0.570 & 650 & 29.90
\end{tabular}

Calculations were made on the Cray YMP computer. The grid-dependency of the solutions was examined by using the two convection schemes, HLPA and HYBRID (central/upwind differencing), and three grids consisting of $50 \times 40$ (grid 1), $86 \times 50$ (grid 2) and $120 \times 80$ (grid 3) points, respectively. The HYBRID scheme that is highly diffusive in the presence of both convective dominance and flow-to-grid skewness has been used here only to highlight the importance of using higher-order accurate schemes. Test results obtained with the RRSAE model at $C_{t}=0.506$ in the BC-case are shown in Fig.2 for the turbulent shear stress $\overline{u v}$-profiles at the downstream location $x / D_{o}=1.875$. It can be seen that the results with HLPA on the coarse grid 1 are already very close to those on the fine grid 3 , while significant differences exist between the corresponding results with HYBRID. The HLPA results on the intermediate grid 2 can be considered as grid-independent because the refinement from the grid 2 to the grid 3 produced differences too small to be seen on the graph. The HYBRID solutions, however, responded to the grid refinement in such a slow manner that they still have not reached the grid-independent stage on the finest grid. The HYBRID scheme is, therefore, inappropriate for computations of confined jets in presence of recirculation. The iterations and CPU-time in minutes required for the calculations with HLPA were 196 and 0.2 on grid 1,640 and 1.4 on grid 2 and 1874 and 9.3 on grid 3. The calculations with HYBRID took about $0.6 \sim 0.8$ of these numbers. The grid 2 and HLPA were used for all subsequent calculations.

Figs. 3 and 4 show the decay of the centerline velocity $U_{o}$, normalized by the mean velocity of the section $U_{m}$, at different $C_{t}$ numbers. The figures clearly reveal the existence of the potential core characterized by the constant $U_{o}$. Beyond the potential core, $U_{o}$ decays quickly, especially at small $C_{t}$ numbers. The experimental data show that the potential core is shorter in the BK-case than in the BC-case, because the former has a larger mixing layer between the jet and the ambient flow at the entrance. This feature is also reflected in the computed results. The RRSAE 
model predicts a longer potential core length than the $K-\varepsilon$ model does, but it is difficult to judge which is better because this length cannot be precisely determined from the first and second experimental points. Overall, both models predict the centerline velocity decay well, though small underpredictions occur in the $K-\varepsilon$ model results at the intermediate and small values of $C_{t}$ in both cases. Figs.5 and 6 show the axial mean velocity profiles at four downstream locations, at the smallest value of $C_{t}$ in each case. In the BC-case, the RRSAE model results are in excellent agreement with the experimental data whereas noticeable discrepancies exist in the $K-\varepsilon$ model results at the last three downstream locations. In the BK-case, the results of RRSAE model are also better than those of the $K-\varepsilon$ model, but the agreement is less satisfactory than in the BC-case. At the location $x / D_{0}=1.25$, the experimental profile shows no constant ambient velocity portion while the calculated profiles by both models still have a vertical plateau around $r / R=0.6$. The comparison at the location $x / D_{0}=2.5$ indicates that the width of the predicted reverse-flow region is somewhat too thin. Comparing the corresponding profiles in the BC-case (Fig.5) which has a much stronger recirculation, the experimental velocity minimum at the last location in Fig.6 seems to be too far away from the duct wall. It is to be noted that the flow is highly perturbed in the recirculation region in which the measurement uncertainty is likely to be greatest.

In order to study the jet spreading, the excess flow rate $Q_{j}$ has been introduced which is defined as follows

$$
Q_{j}=2 \pi \int_{0}^{r_{2}}\left(U-U_{1}\right) r d r
$$

where $U_{1}$ is the ambient velocity (Fig.1). In the recirculation region, the ambient velocity has no physical meaning and is defined as the minimum velocity (having negative value) for analytical convenience. Figs.7 and 8 show the variation of the excess flow rate $Q_{j}$ with $x$ and $C_{t}$. As a consequence of the turbulent entrainment, the excess flow rate increases first, passes through a maximum and then decreases in case of recirculation which corresponds to $Q_{j} / Q \geq 1$. This variation becomes more pronounced as $C_{t}$ decreases. The calculations agree well with the experiments at large $C_{t}$, but the agreement deteriorates as recirculation intensifies. At small $C_{t}$ $(<0.6)$, the calculations overpredict the maximum excess flow rate in the BC-case, while the opposite happens in the BK-case. It should be pointed out that the excess flow rate, due to its definition, is a quantity that is highly sensitive to errors in the velocity profiles so that a small change in $U_{1}$, especially in the recirculation zone, will result in a large difference in $Q_{j}$. Regarding the comparison between the two models, the RRSAE model clearly performs better than does the $K-\varepsilon$ model.

The separation and reattachment points are given in Table 4 . They are the 
locations where $Q_{j} / Q=1$ in Figs.7 and 8 . In the BK-case, only a range of $3.4 \sim 3.8 D_{o}$ was given experimentally for the reattachment point due to the high unsteadiness of the recirculating bubbles. The experiments in both the BC- and BK-cases revealed that as $C_{t}$ decreased, the separation points moved upstream while the reattchment points remained basically unchanged. The RRSAE model captures this feature well and predicts the locations of the recirculation bubbles better than the $K-\varepsilon$ model.

Table 4. Separation and reattachment points $\left(x_{\bullet} / D_{0}, x_{r} / D_{0}\right)$

\begin{tabular}{ccccc}
\hline case & $C_{t}$ & experiment & $K-\varepsilon$ model & RRSAE model \\
\hline BC & 0.506 & $1.70,3.07$ & $1.37,2.7$ & $1.6,3.03$ \\
\cline { 2 - 5 } & 0.152 & $0.45,3.07$ & $0.40,2.95$ & $0.5,3.15$ \\
\hline BK & 0.775 & $2.50,3.4 \sim 3.8$ & $1.82,3.17$ & $2.15,3.79$ \\
\cline { 2 - 5 } & 0.590 & $1.50,3.4 \sim 3.8$ & $1.43,3.22$ & $1.45,3.81$ \\
\hline
\end{tabular}

Figs. 9 and 10 show the variation of pressure coefficients along the duct walls. Here, $C_{p}$ is defined by

$$
C_{p}=\frac{\Delta p-\rho U_{a}^{2} / 2}{\rho U_{J}^{2} / 2}
$$

and $\Delta p$ is the pressure difference between the location $x$ and the entrance. The pressure gradient is governed by the jet entrainment, the contraction and expansion of the flow caused by recirculating eddies as well as the geometry of the duct. As shown in Kian (1981), the entrainment and the divergence of the duct can only produce a maximum pressure difference equal to $\rho U_{a}^{2} / 2$, while the pressure difference created by the divergence of streamlines in the downstream part of the recirculating bubble is of the order of

$$
\frac{\Delta p}{\rho U_{a}^{2}} \sim\left(1+K_{c}\right)\left(\frac{R_{o}}{R_{c}}\right)^{4}
$$

where the coefficient $K_{\mathrm{c}}$ is a positive quantity defined by the flow velocity, and $R_{c}$ is the minimum radius of the contracted stream. Therefore, the pressure rise in the recirculating zone depends on the width of the recirculating bubble and can be much larger than $\rho U_{a}^{2} / 2$. This explains the variation of $C_{p}$ with $C_{t}$ seen in Figs.9 and 10 . Regarding the comparison between predictions and experiments, it can be seen that although both models predict practically the same total pressure rise which is in very good agreement with the measurements (Fig.9), the RRSAE model captures the location where the pressure starts to shoot up much better than the $K-\varepsilon$ model at all the $C_{t}$ numbers in both the cases. This location corresponds to the abscissa of the maximum excess flow rate shown in Figs.7 and 8. 
Detailed experimental data for the turbulent stresses $\overline{u u}, \overline{v v}$ and $\overline{u v}$ are available only in the BK-case at $C_{t}=0.59$. The computed and measured radial profiles of these quantities at four downstream locations are compared in Figs.11-13. With regard to the turbulent normal stresses shown in Figs.11 and 12, the experimental data are basically followed by the results of both the RRSAE model and the $K-\varepsilon$ model, with the former predicting more anisotropy than the latter. The experimental data at $x / D_{o}=2.5$ are seen to exhibit a different trend for both $\bar{u} \bar{u}$ and $\bar{v} \bar{v}$, which may possibly be due to measurement errors. The flow visualization in the experiment indicated that the global flow pattern was highly unsteady in the presence of recirculation. With due regard to flow complexities and measurement difficulties, the agreement between the predictions and measurements seen in Figs.11 and 12 should be considered as reasonably good, but it is difficult to judge which model performs better for the turbulent normal stresses, overall. For the turbulent shear stress $\overline{u v}$ shown in Fig.13, the results obtained with the RRSAE model are clearly better than those with the $K-\varepsilon$ model for all the locations considered. The large discrepancy seen at $x / D_{0}=2.5$ is partially due to the underprediction of the width of the backflow region and partially due to the experimental uncertainty, as evidenced by the fact that in the experimental data, the change in sign of the shear stress profile occurs much further away from the duct wall than the velocity minimum.

\section{Concluding Remarks}

A numerical study has been conducted on axisymmetric confined jets in two ducts with and without divergence. The focus of the study was on the performance of the two turbulence models under various flow conditions ranging from no to strong recirculation. The RRSAE model is new, and its capability to predict this type of flows needs to be tested. The $K-\varepsilon$ model was applied to the same problem before, but we believe that a re-assessment is necessary because the previous studies were made on an inaccurate numerical basis (first-order differencing together with very coarse grids). This study has shown that the confined jet calculations are very sensitive to the convective approximation of the transport equations. In order to achieve a numerically credible solution without involving excessive grid points, higher-order differencing schemes have to be adopted. In this regard, the second-order accurate HLPA scheme behaves quite well and its solution is virtually the same as those of third-order accurate SMART and SHARP schemes (Zhu, 1992), all three being of oscillation-free nature. Therefore, it can be concluded with a sufficient degree of confidence that the present calculations reflect the real predictive capability of the 
models for the confined jets.

The calculations have been extensively compared with the experiments. The comparison clearly shows the superiority of the RRSAE model over the $K-\varepsilon$ model under all the circumstances considered. Specifically, the former performs much better than the latter in the predictions of the pressure distribution, the separation and reattachment points of recirculation region, the excess flow rate, the jet expansion and the turbulent shear stress. Only for the maximum reverse flow rate, an important parameter to characterize the performance of combustion chambers, does the RRSAE model result in little improvement. Since this quantity is directly related to the flow near the wall in the recirculation region, the use of the wall function may constitute a source of error and also measurement errors should not be excluded in this highly perturbed region. Overall, the RRSAE model is shown to be able to predict confined jets with an accuracy sufficient for engineering applications.

\section{References}

1. Barchilon, M., and Curtet, R., 1964, "Some details of the structure of an axisymmetric confined jet with backflow," Journal of Basic Engineering, Vol.86, pp.777-787.

2. Barchilon, M., 1969, "Contribution à l'étude des courants de retour engendrés par un jet confiné," Publications Scientifiques et Techniques du Ministère de l'Air, Paris.

3. Binder, G., and Kian, K., 1983, "Confined jets in a diverging duct," Proceedings, Turbulent Shear Flows 4, Karlsruhe, pp.7.18-7.23.

4. Craya, A., and Curtet, R., 1955, "Sur l'évolution d'un jet en espace confiné," Comptes-Rendus de l'Académie des Sciences, Paris, Vol.241, pp.621-622.

5. Curtet, R., 1960, "Sur l'écoulement d'un jet entre parois," Publications Scientifiques et Techniques du Ministère de l'Air, No.359, Paris.

6. Gosman, A.D., Khalil, E.E., and Whitelaw, J.H., 1979, "The calculation of two-dimensional turbulent recirculating flows," Turbulent Shear Flows 1, edited by F. Durst, B.E. Launder, F.W. Schmidt, and J.H. Whitelaw, SpringerVerlag, pp.237-255.

7. Habib, M.A., and Whitelaw, J.H., 1979, "Velocity characteristics of a confined coaxial jet," Journal of Fluids Engineering, Vol.101, pp.521-529.

8. Habib, M.A., and Whitelaw, J.H., 1981, "Calculations of confined coaxial-jet flows," Proceedings, Turbulent Shear Flows 3, University of California, Davis, pp.5.20-5.26. 
9. Hill, P.G., 1967, "Incompressible jet mixing in converging-diverging axisymmetric ducts," Journal of Basic Engineering, Vol.89, pp.210-220.

10. Hill, B.J., 1973, "Two-dimensional analysis of flow in jet pumps," Journal of the Hydraulics Division, ASCE, Vol.99, HY7, pp.1009-1026.

11. Jones, W.P., and Marquis, A.J., 1985, "Calculation of axisymmetric recirculating flows with a second order turbulent model," Proceedings, Turbulent Shear Flows 5, Cornell University, Ithaca, pp.20.1-20.6.

12. Khalil, E.E., Spalding, D.B., and Whitelaw, J.H., 1975, "The calculation of local flow properties in two-dimensional furnaces," International Journal of Heat and Mass Transfer, Vol.18, pp.775-791.

13. Kian, K., 1981, Jets confinés dans un divergent, Doctoral dissertation, l'Université Scientifique et Médicale de Grenoble et l'Institut National Polytechnique de Grenoble, France.

14. Launder, B.E., and Spalding, D.B., 1974, "The numerical computation of turbulent flows," Computer Methods in Applied Mechanics and Engineering, Vol.3, pp.269-289.

15. Lumley, J.L., 1978, "Computational modeling of turbulent flows," Adv. Appl. Mech., Vol.18, pp.124-176.

16. Mikhail, S., 1960, "Mixing of coaxial streams inside a closed conduit," Journal of Mechanical Engineering Science, Vol.2, pp.59-68.

17. Rajaratnam, N., 1976, Turbulent Jets, Elsevier Scientific Publishing Company.

18. Rajaratnam, N., and Pani, B.S., 1972, "Turbulent compound annular shear layers," Journal of the Hydraulics Division, ASCE, Vol.98, HY7, pp.1101-1115.

19. Razinsky, E., and Brighton, J.A., 1971, "Confined jet mixing for nonseparating conditions," Journal of Basic Engineering, Vol.93, pp.333-349.

20. Razinsky, E., and Brighton, J.A., 1972, "A theoretical model for nonseparated mixing of a confined jet," Journal of Basic Engineering, Vol.94, pp.551-558.

21. Rhie, C.M., and Chow, W.L., 1983, "A numerical study of the turbulent flow past an isolated airfoil with trailing edge separation," AIAA Journal, Vol.21, pp.1525-1532.

22. Rodi, W., Majumdar, S., and Schönung, B., 1989, "Finite-volume method for two-dimensional incompressible flows with complex boundaries," Computer Methods in Applied Mechanics and Engineering, Vol.75, pp.369-392.

23. Schumann, U., 1977, "Realizability of Reynolds stress turbulence models," Physics of Fluids, Vol.20, pp.721-725.

24. Shih, T.-H., and Lumley, J.L., 1993, "Remarks on turbulent constitutive relations," NASA TM 106116. 
25. Shih, T.-H., Zhu, J., and Lumley, J.L., 1993, "A realizable Reynolds stress algebraic equation model," NASA TM 105993.

26. Spalding, D.B., 1972, "A novel finite difference formulation for differential expressions involving both first and second derivatives," International Journal for Numerical Methods in Engineering, Vol.4, pp.551-559.

27. Stone, H.L., 1968, "Iterative solution of implicit approximations of multidimensional partial differential equations," SIAM J. Num. Anal, Vol.5, pp.530558.

28. Van Doormal, J.P., and Raithby, G.D., 1984, "Enhancements of the SIMPLE method for predicting incompressible fluid flows," Numerical Heat Transfer, Vol.7, pp.147-163.

29. Zhu, J., 1986, "Calcul des jets turbulents confinés avec recirculation," Doctoral dissertation, l'Institut National Polytechnique de Grenoble, France.

30. Zhu, J., Binder, G., and Kueny, J.L., 1987, "Improved predictions of confined jets with a parabolic computation of the entrance region," AIAA Journal, Vol.25, pp.1141-1142.

31. Zhu, J., 1991a, "A low diffusive and oscillation-free convection scheme", Communications in Applied Numerical Methods., Vol.7, pp.225-232.

32. Zhu, J., 1991b, "FAST-2D: A computer program for numerical simulation of two-dimensional incompressible flows with complex boundaries", Rept. No.690, Institute for Hydromechanics, University of Karlsruhe.

33. Zhu, J., and Rodi, W., 1992, "Computation of axisymmetric confined jets in a diffuser," International Journal for Numerical Methods in Fluids, Vol.14, pp.241-251.

34. Zhu, J., 1992, "On the higher-order bounded discretization schemes for finitevolume computations of incompressible flows," Computer Methods in Applied Mechanics and Engineering, Vol.98, pp.345-360.

35. Zhu, J., and Shih, T.-H., 1993, "Calculations of turbulent separated flows," NASA TM 106154. 


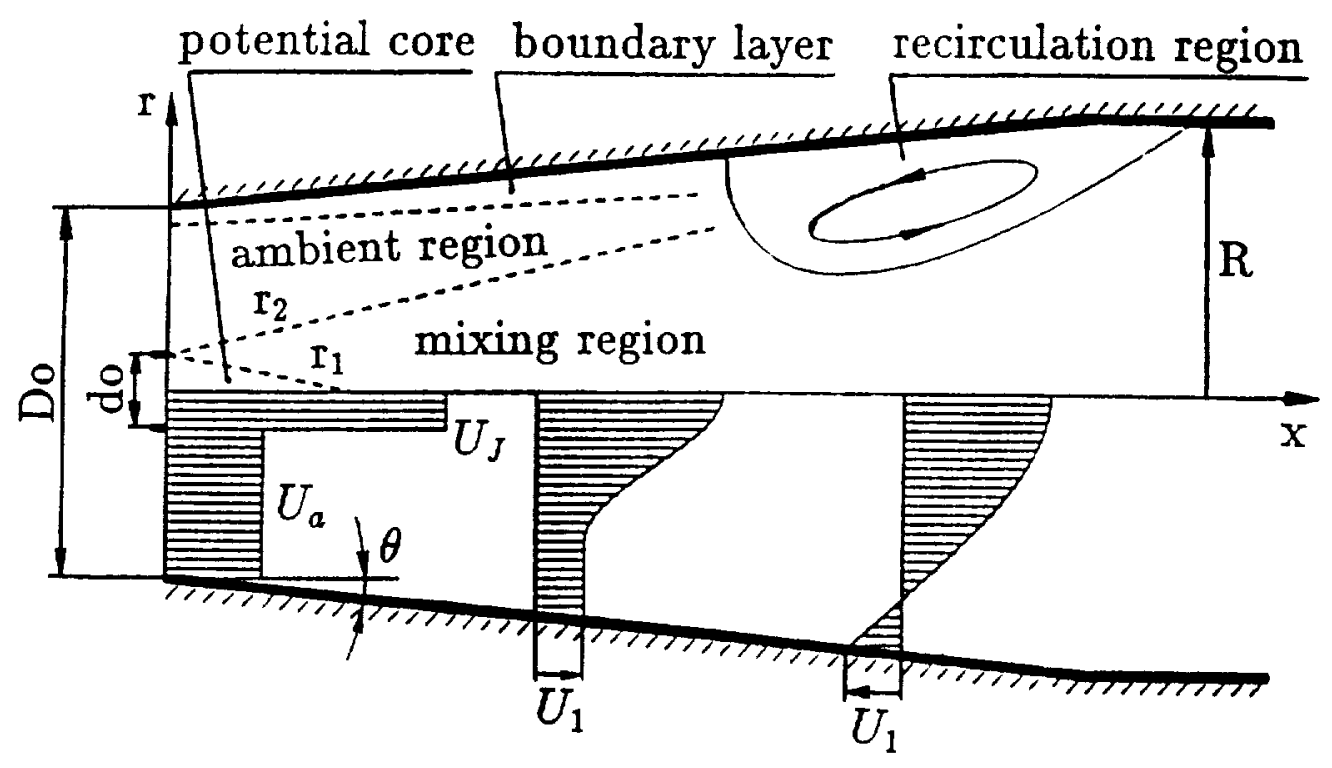

Figure 1. Flow configuration and notations

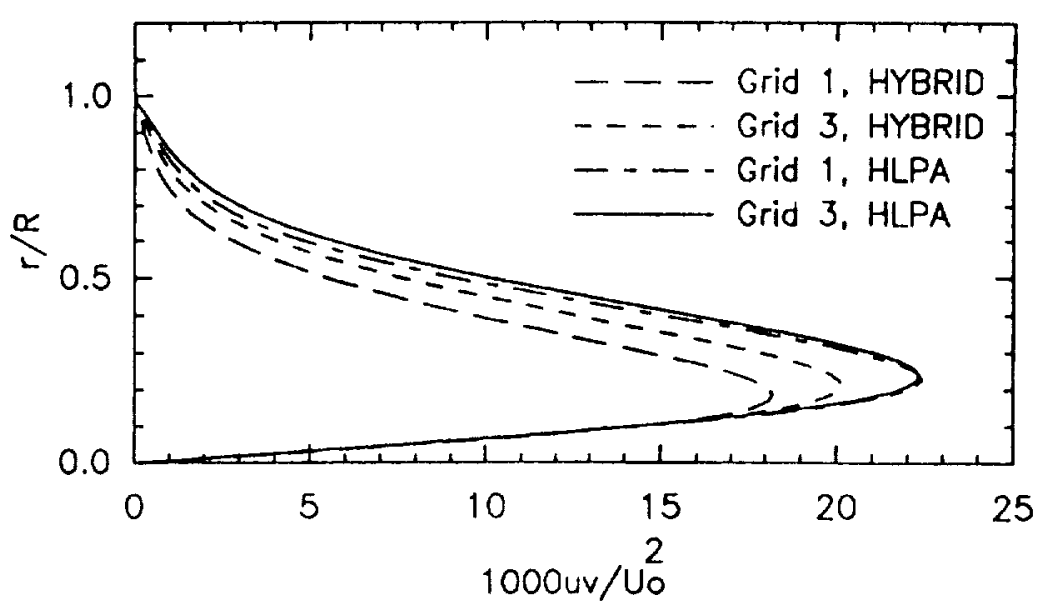

Figure 2. Numerical accuracy test at $C_{t}=0.506$ in the BC-case 


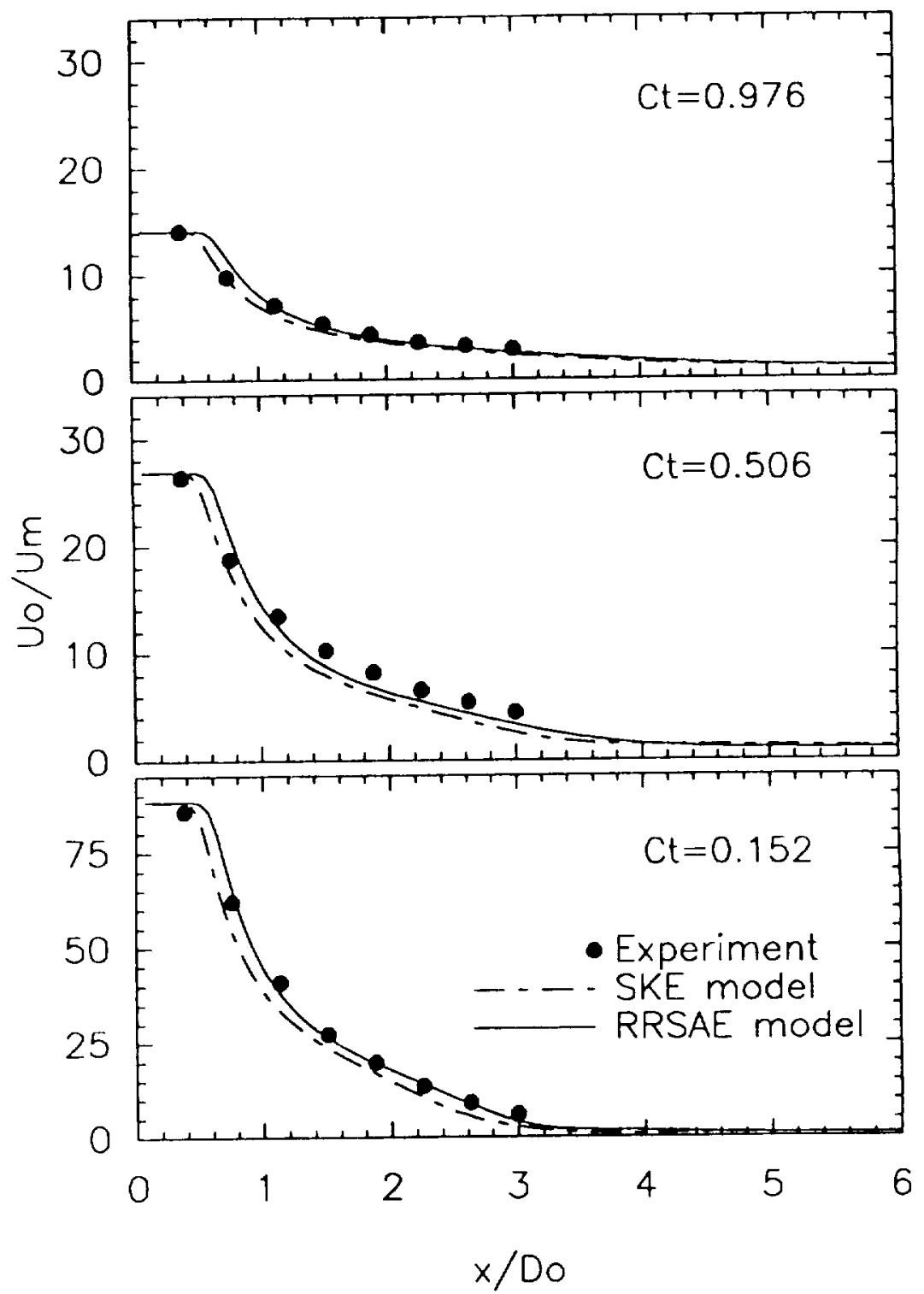

Figure 3. Centerline velocity decay in the $\mathrm{BC}$-case 


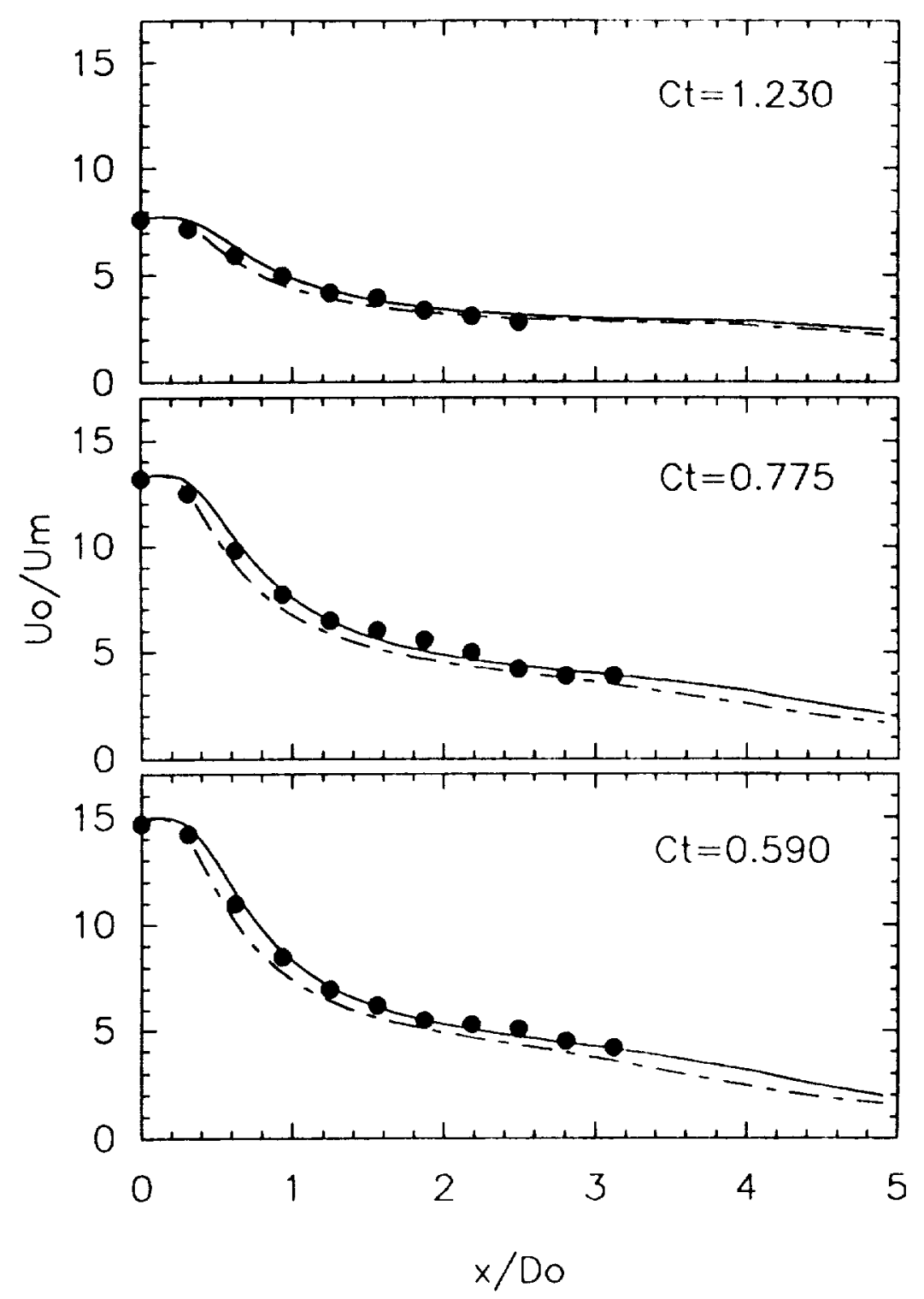

Figure 4. Centerline velocity decay in the BK-case (Notation as in figure 3) 


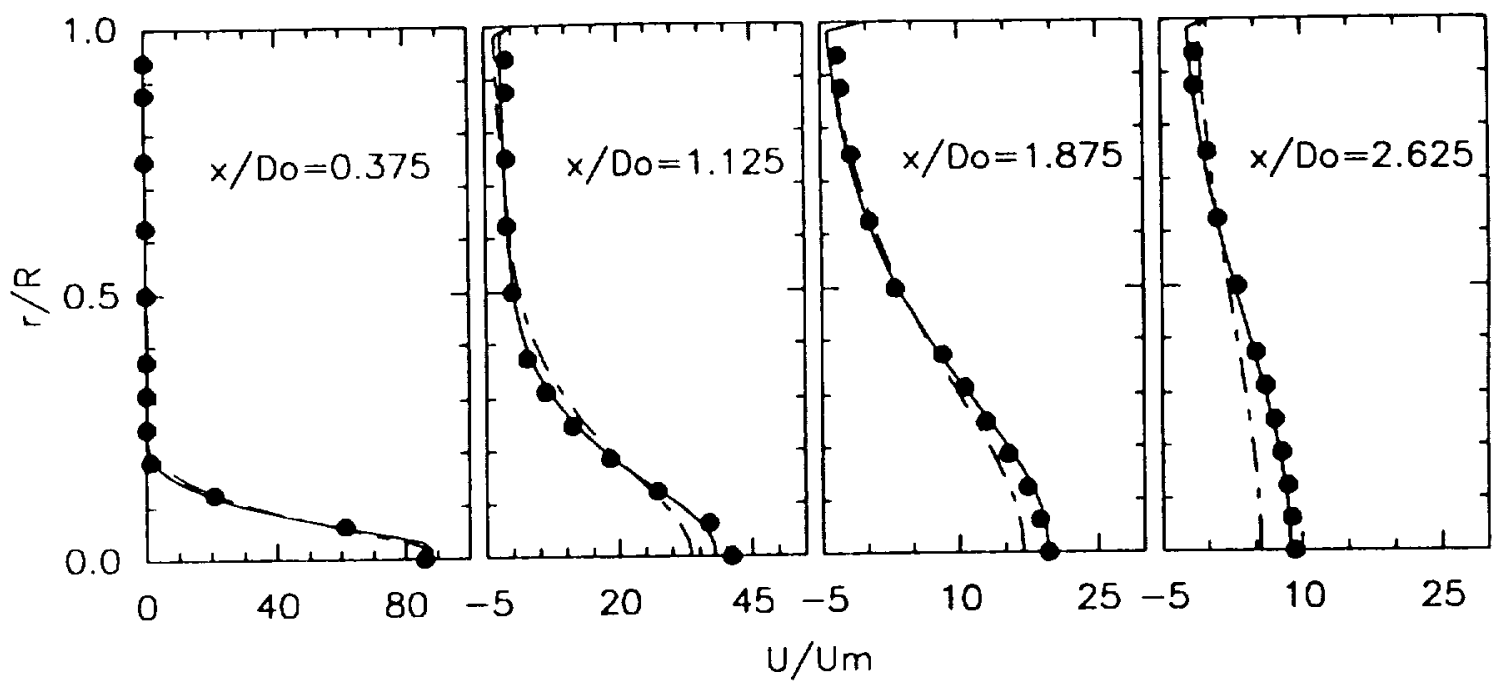

Figure 5. Axial mean velocity profiles at $C_{t}=0.152$ in the BC-case (Notation as in figure 3 )

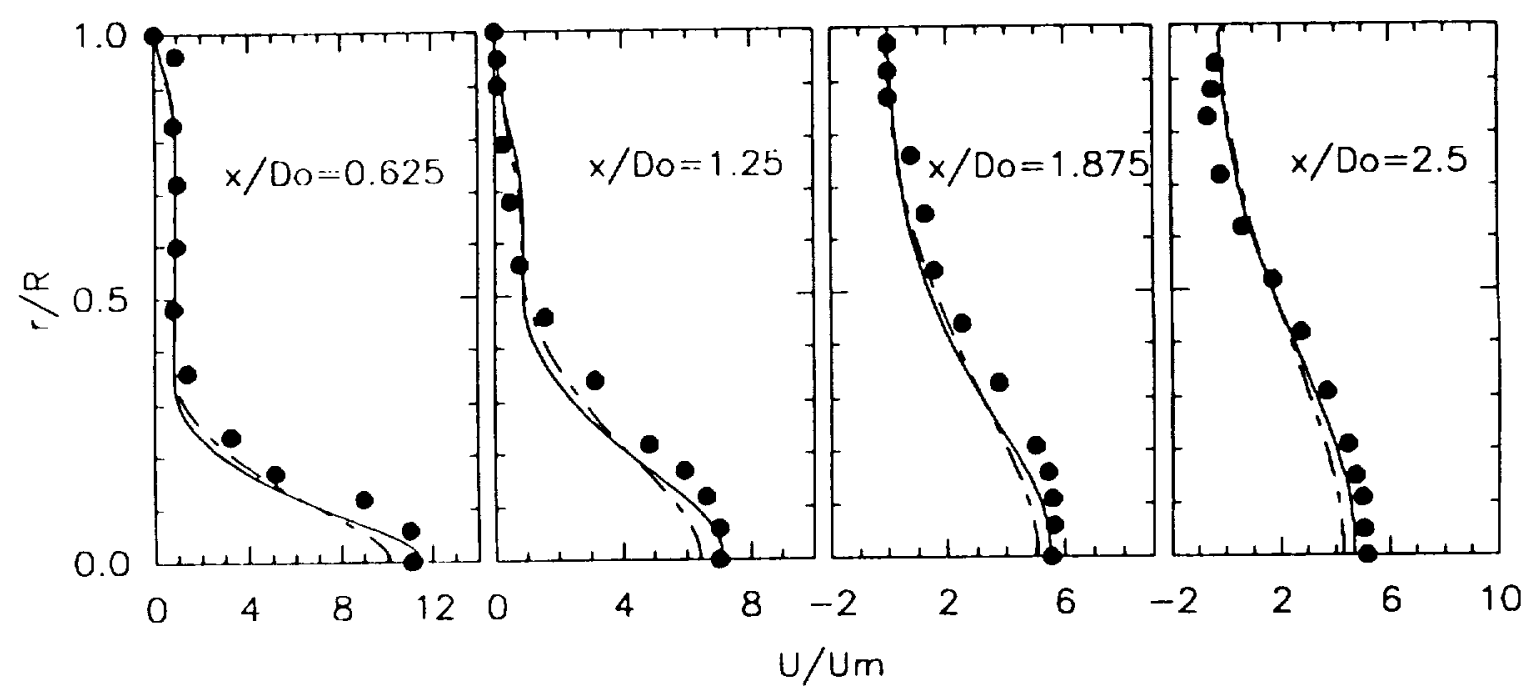

Figure 6. Axial mean velocity profiles at $C_{t}=0.59$ in the BK-case (Notation as in figure 3 ) 


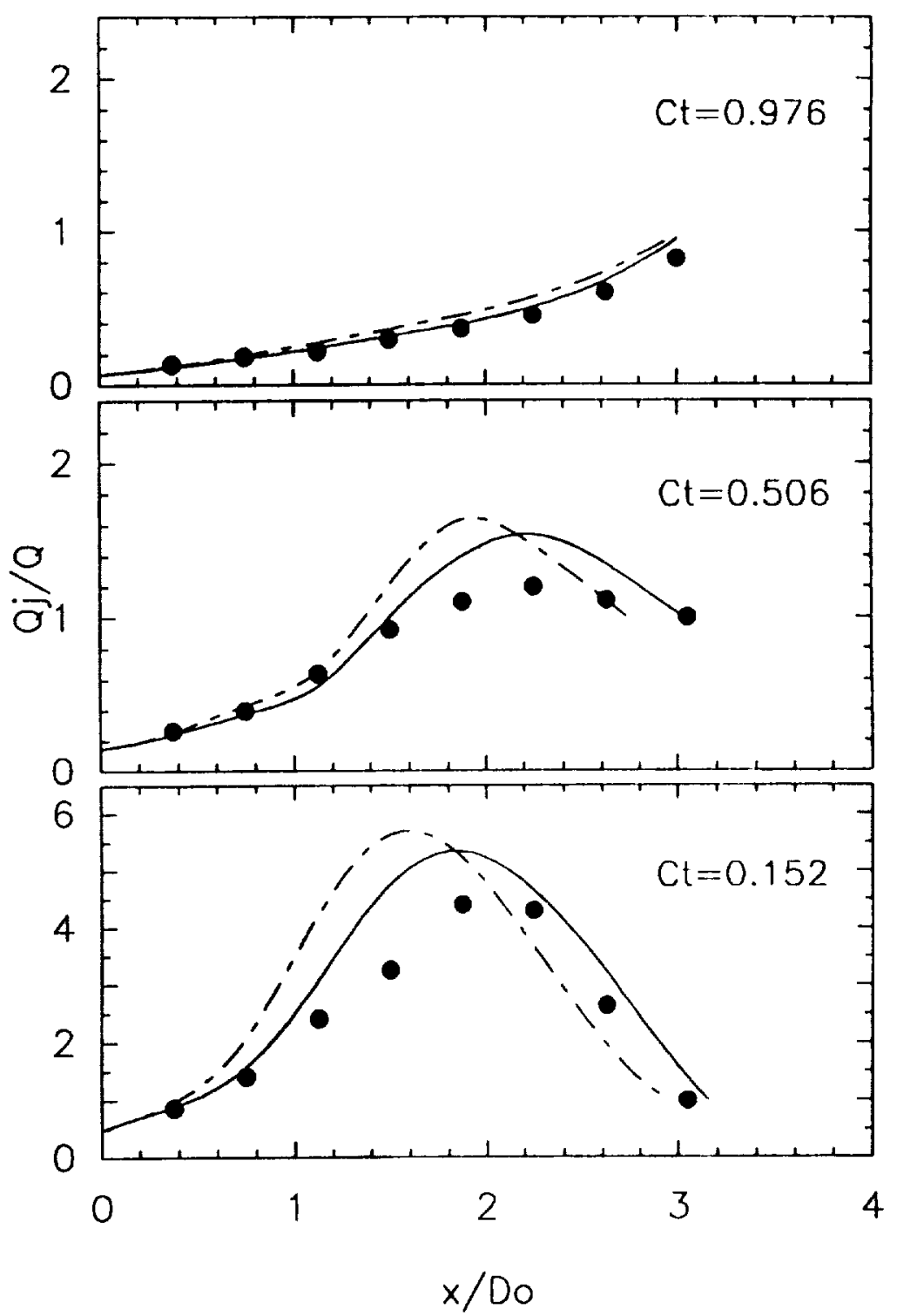

Figure 7. Excess flow rate in the BC-case (Notation as in figure 3) 


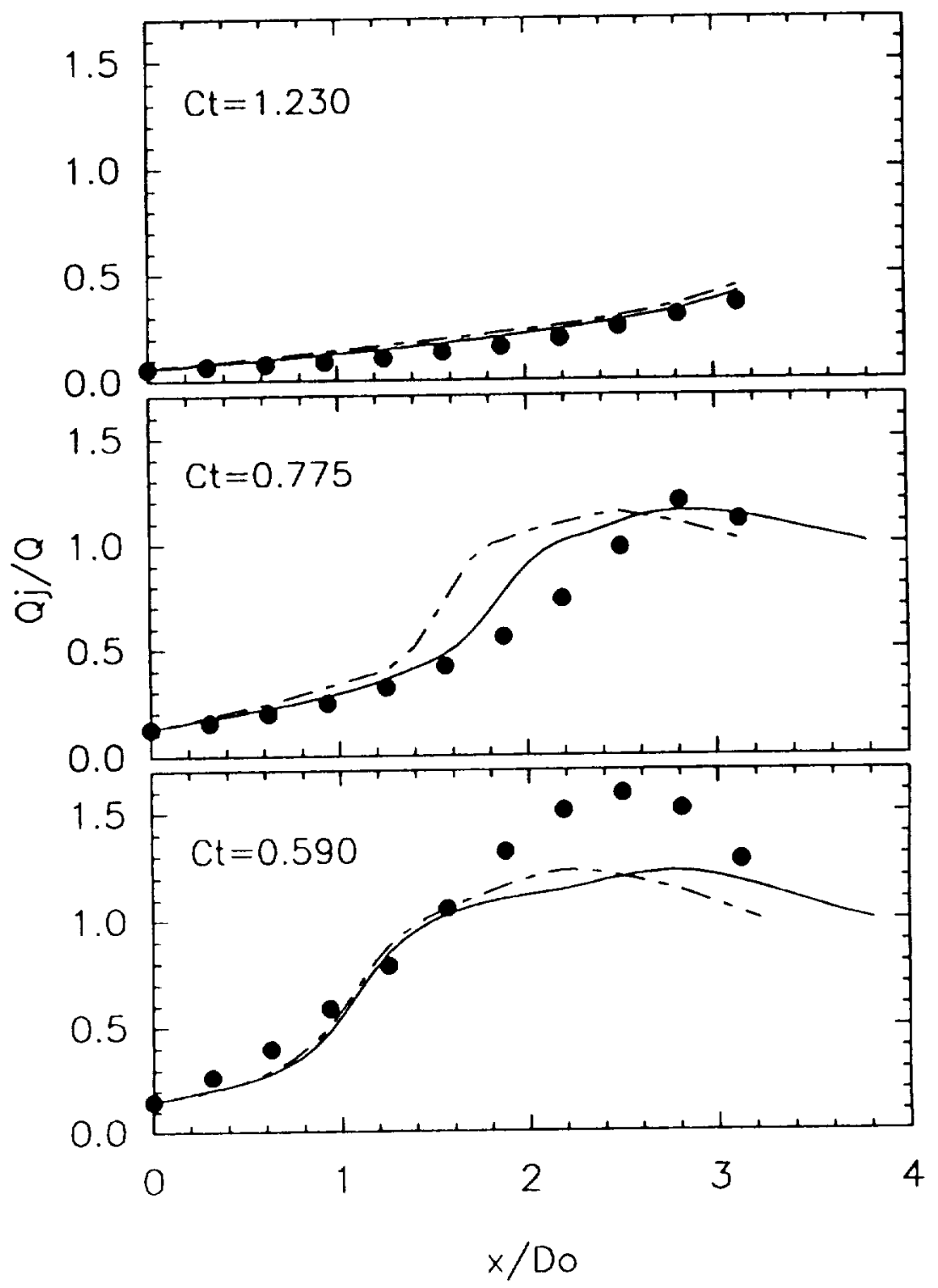

Figure 8. Excess flow rate in the BK-case (Notation as in figure 3) 


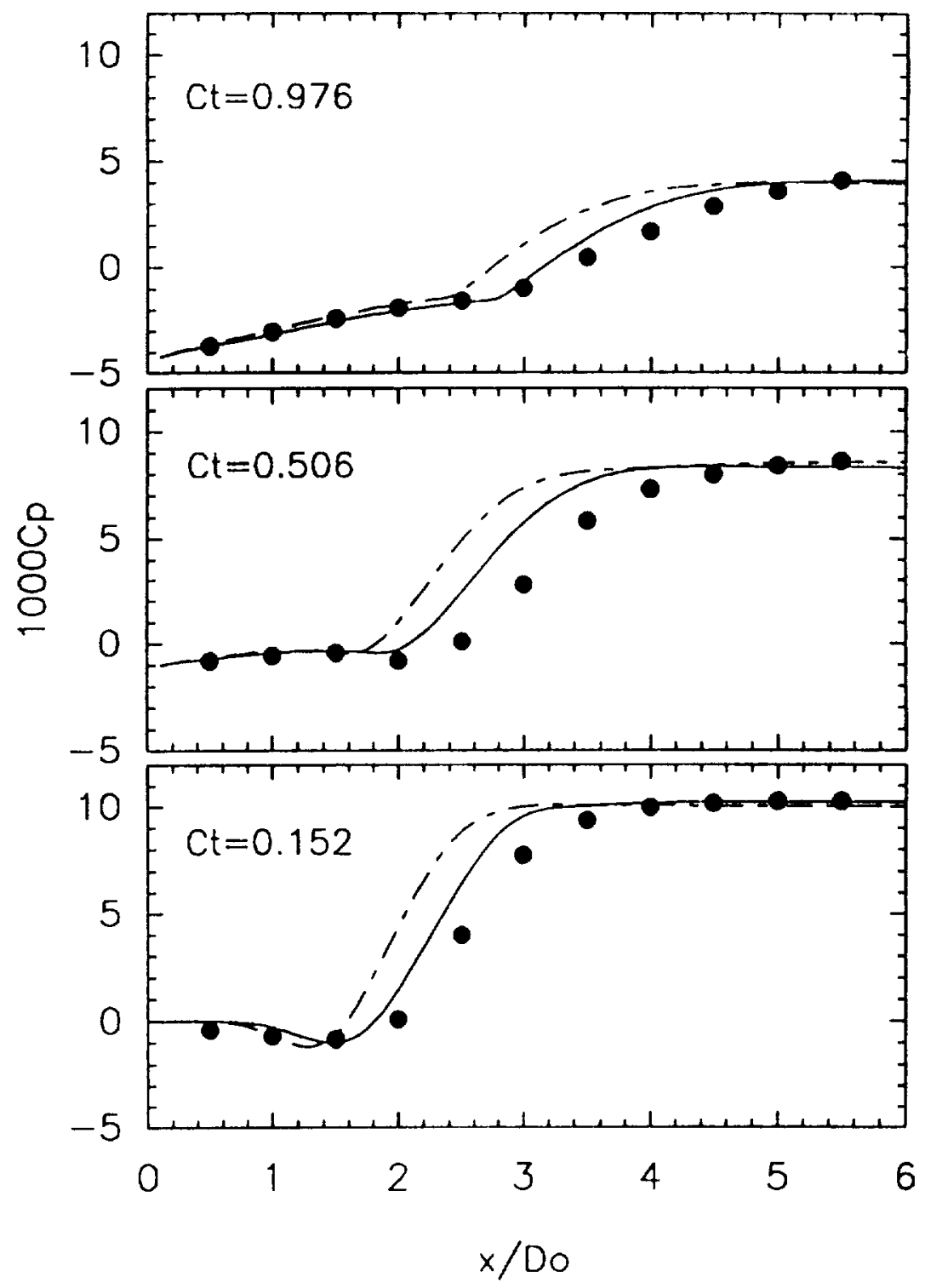

Figure 9. Pressure coefficient along the duct wall in the BC-case (Notation as in figure 3 ) 


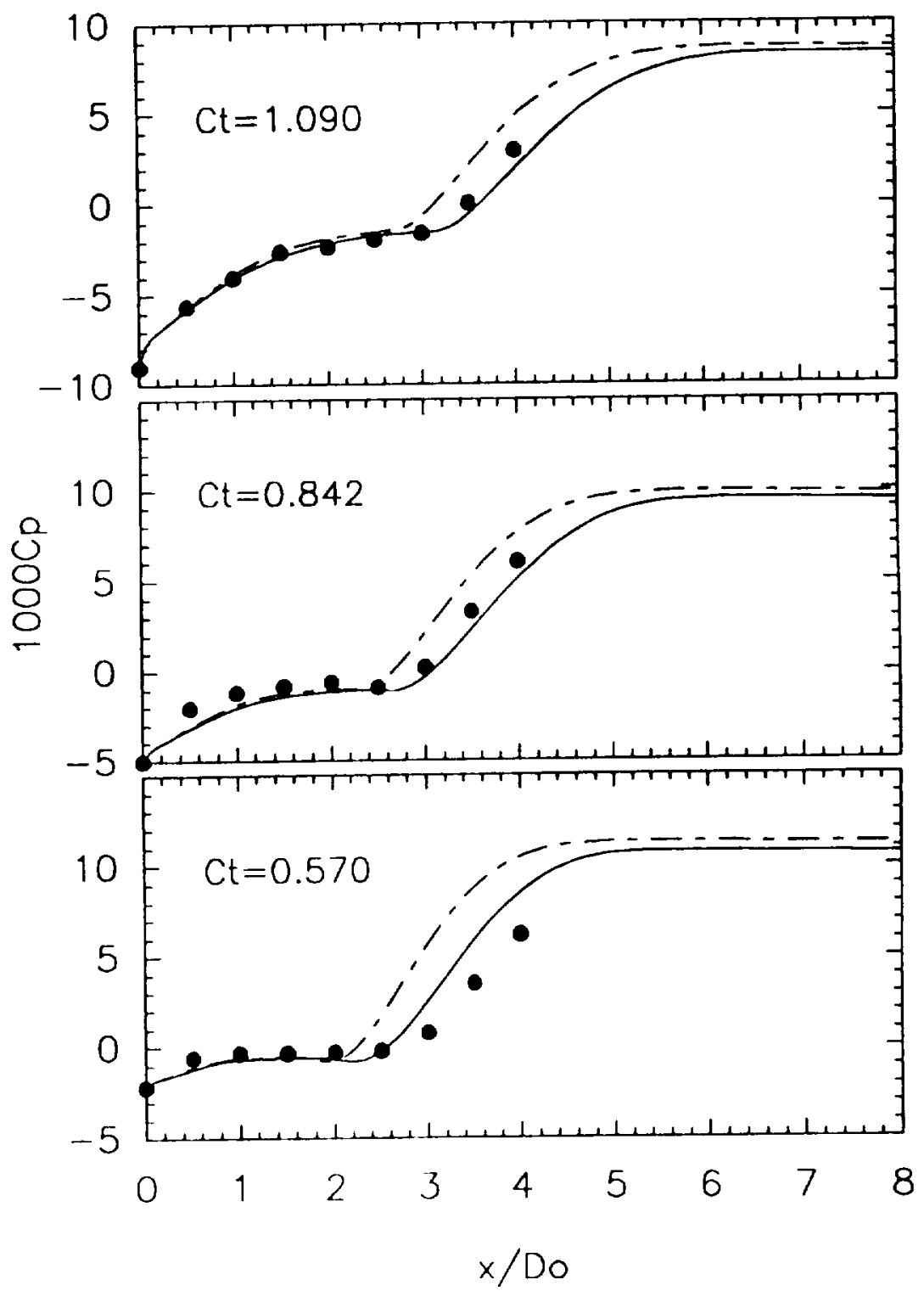

Figure 10. Pressure coeflicient along the duct wall in the BK-case (Notation as in figure 3 ) 


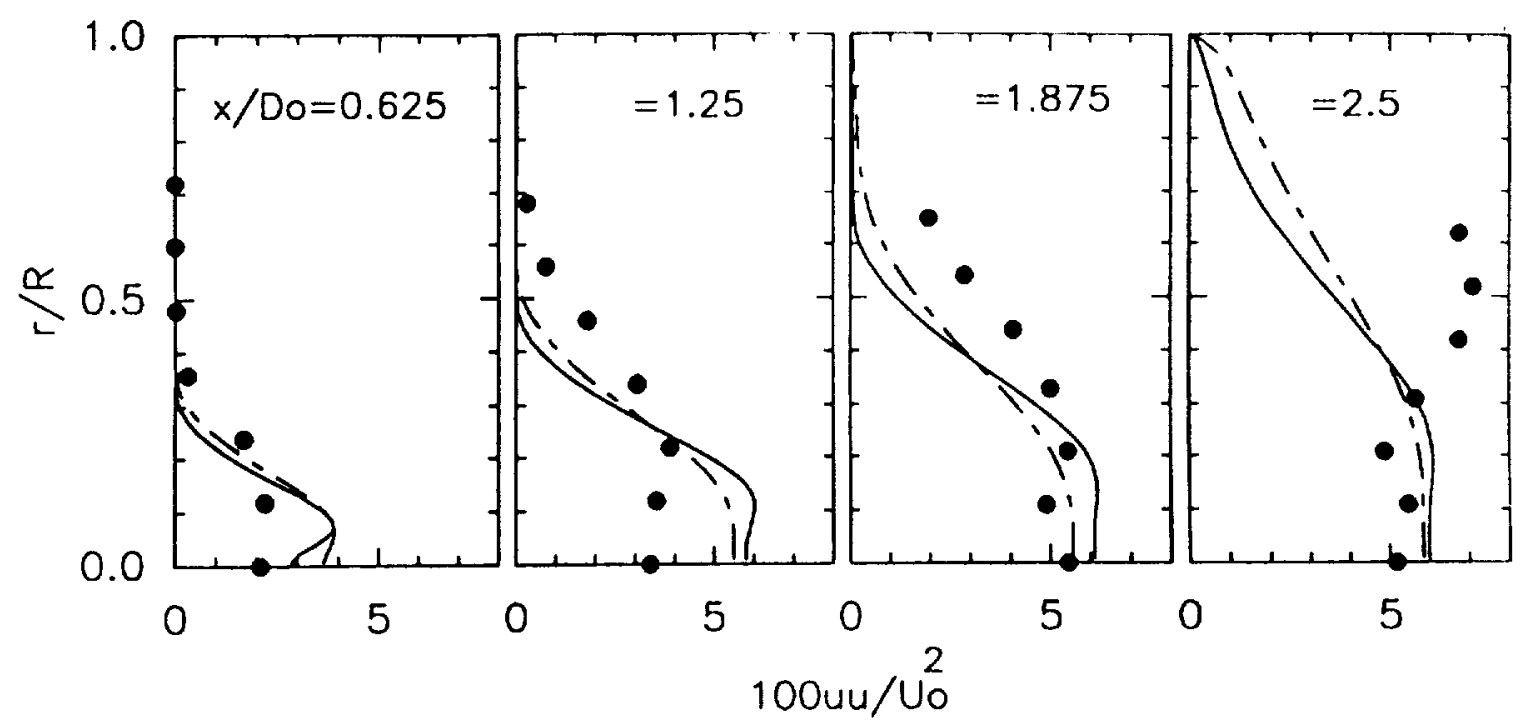

Figure 11. Turbulent normal stress $\bar{u} u$-profiles at $C_{t}=0.59$ in the BK-case (Notation as in figure 3 )

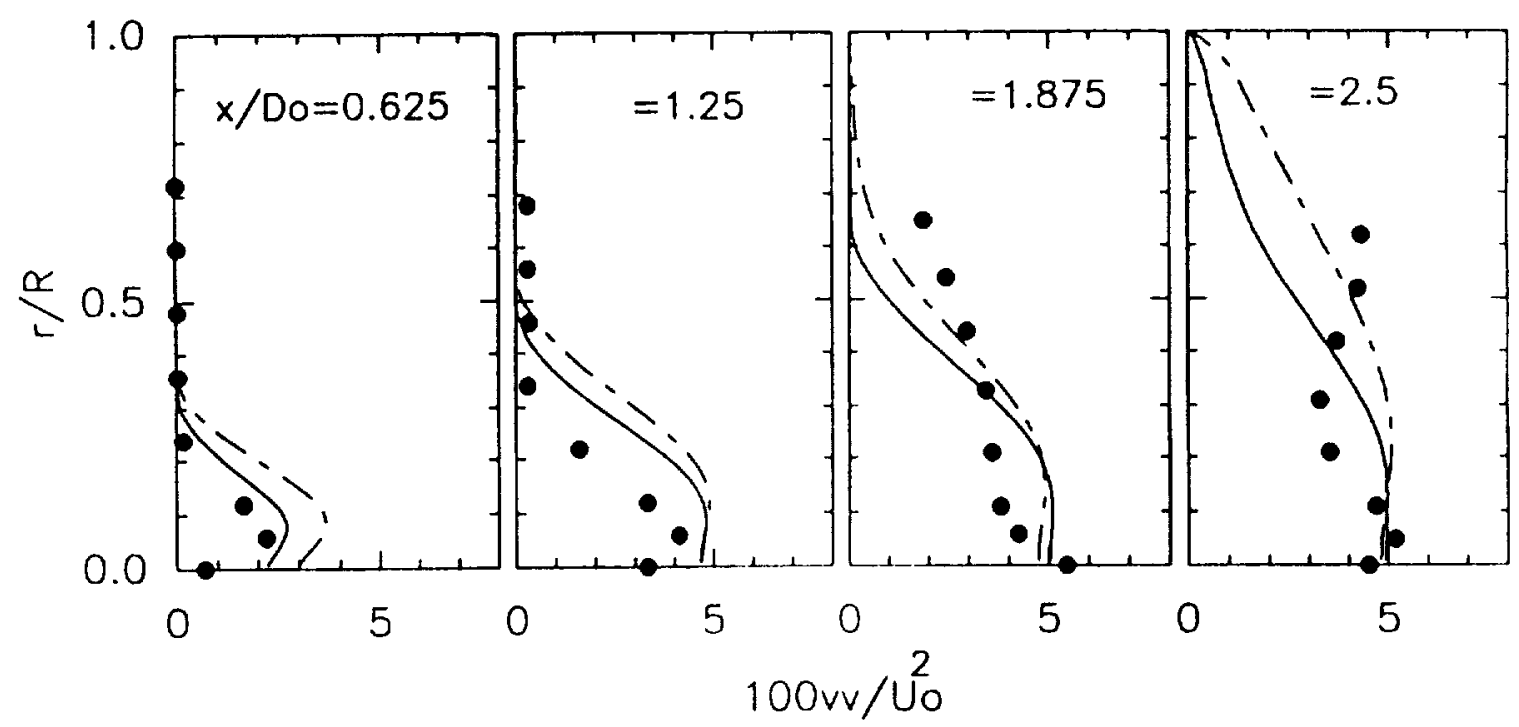

Figure 12. Turbulent normal stress $\bar{v} \bar{v}$-profiles at $C_{t}=0.59$ in the BK-case (Notation as in figure 3 ) 


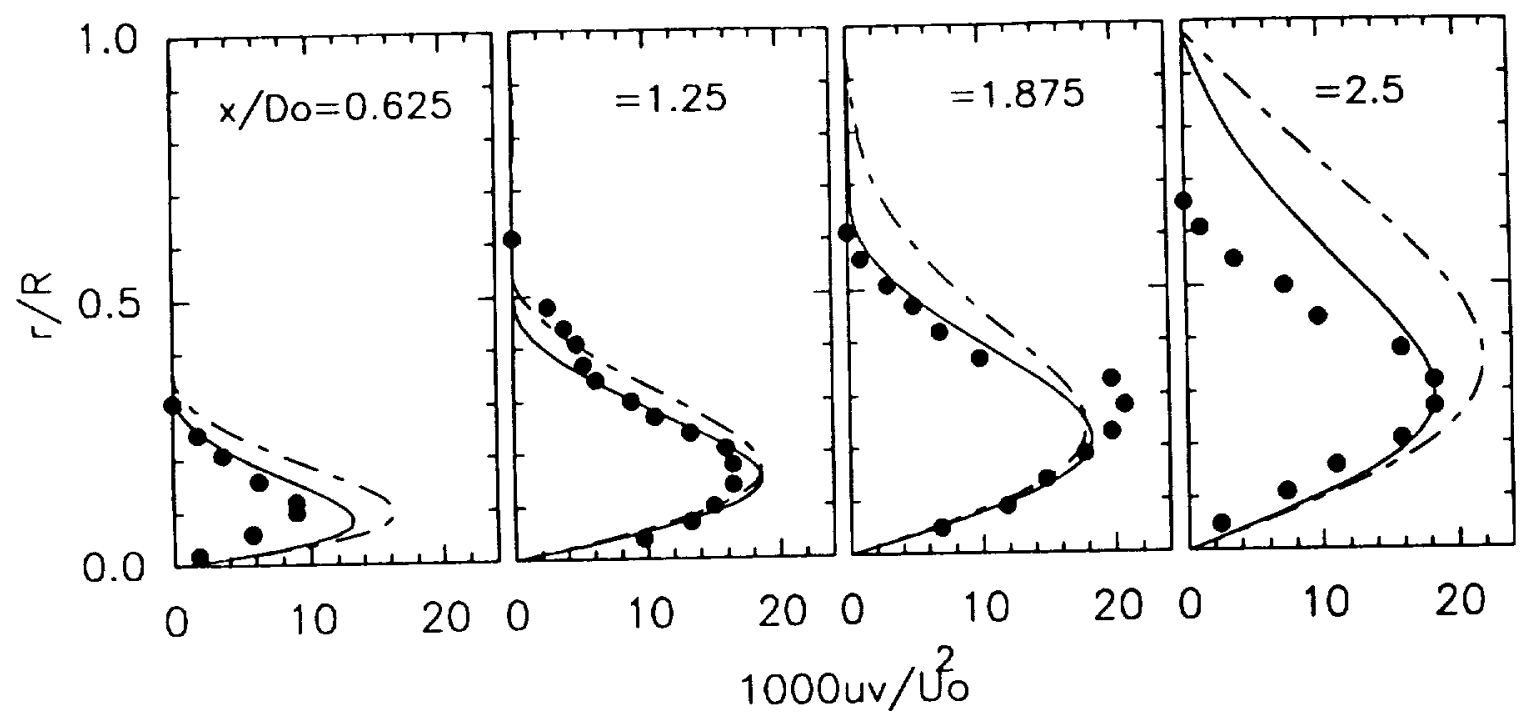

Figure 13. Turbulent shear stress $\bar{u} \bar{v}$-profiles at $C_{t}=0.59$ in the BK-case (Notation as in figure 3 ) 


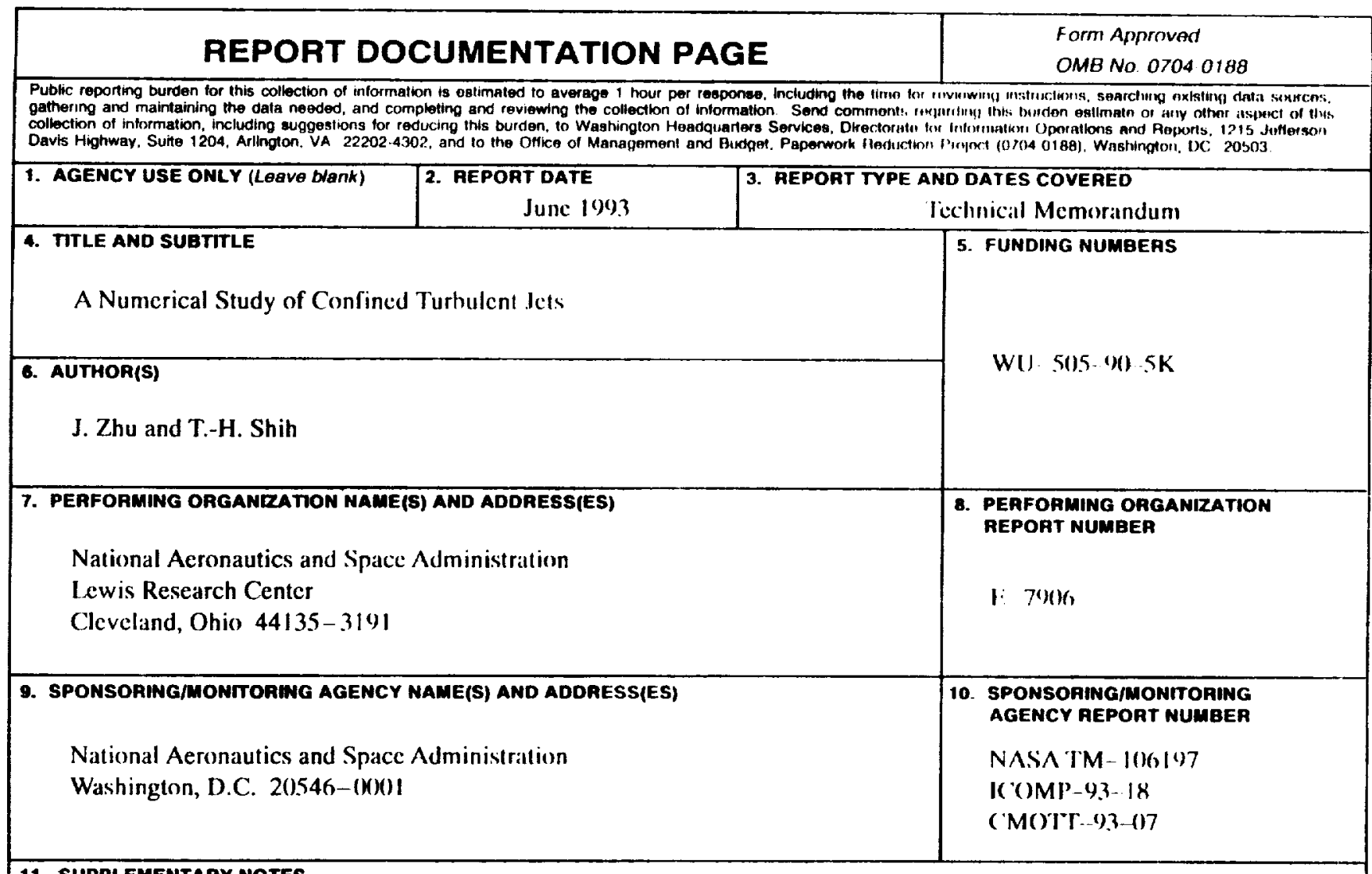

\section{SUPPLEMENTARY NOTES}

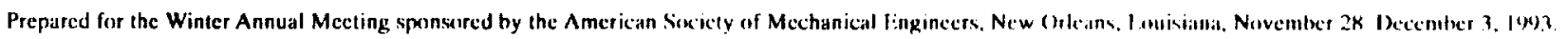

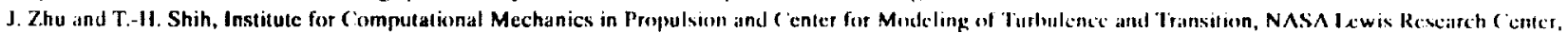

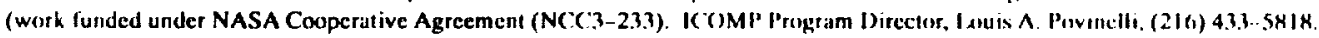

12a. DISTRIBUTION/AVALABUITY STATEMENT 12b. DISTRIBUTION CODE

Unclassified - Unlimited

Subject Category 34

13. ABSTRACT (Maximum 200 words)

A numerical investigation is reported of turhulent incompressible jets confined in two ducts, one cylindrical and the other conical with a $5^{\circ}$ divergence. In cach ease, three ('ritya-c urtel numbers are considered which correspond. respectively, to flow situations with no, moderate and strong recirculation. T'urbulence closite is achicved by using the K-E model and a recently proposed realizahle Reynolds stress algebraic cquation moctel that relates the Reynolds stresses explicitly to the quadratic terms of the mean velocily gradients and ensures the positiveness of each conmponent of the turbulent kinetic energy. Calculations are carried oul with a finite-volume procedure using boundary-fitted curvilinear co-ordinates. A second-order accurate, bounded convection scheme and sufficicntly fine grids are used to prevent the solutions from being contaminated by numcrical diffusion. The calculated results are compared extensively with the available experimental data. It is shown that the numerical methods presented are capable of capturing the essential flow features observed in the experiments and that the rcalizable Reynolds stress algebraic cquation model performs much better than the K-E model for this clatss of flows of great practical importance.

14. SUBJECT TERMS

Confined jets; Turbulence models; Recirculation

\begin{tabular}{|c|c|}
\hline 17. SECURITY CLASSIFICATION & 18. SECUAITY CLASSIFICATION \\
OF REPORT & OF THIS PAGE \\
Unclassified & Unclassificd
\end{tabular}

NSN 7540-01-280-5500

\begin{tabular}{|c|c|}
\hline & $\begin{array}{c}\text { 15. NULAER OF PAGES } \\
27\end{array}$ \\
\hline & $\begin{array}{l}\text { 16. PAICE COODE } \\
\text { AO3 }\end{array}$ \\
\hline $\begin{array}{l}\text { 19. SECUATTY CLASSIFICATION } \\
\text { OF ABSTRACT } \\
\text { Unclassificd }\end{array}$ & 20. LIMITATION OF ABSTRACT \\
\hline
\end{tabular}

